\title{
[FEPAH $]^{+}$COMPLEXES AND [FEXPAH $]^{+}$CLUSTERS IN THE INTERSTELLAR MEDIUM: STABILITY AND SPECTROSCOPY
}

\author{
A. Simon ${ }^{1,2}$, M. Rapacioli ${ }^{1,2}$, F. Spiegelman ${ }^{1,2}$ and C. Joblin ${ }^{3,4}$
}

\begin{abstract}
The relevance of organometallic complexes in the chemistry of the interstellar medium (ISM) was first suggested in the early 90s. This initial proposal has recently been re-considered in the light of new astronomical data, benefiting from pioneering experimental techniques and theoretical methods. In this article, a review of recent theoretical and experimental results obtained for PAHs coordinated to Fe atoms is presented, focusing on: -(i)- the IR spectra of $[\mathrm{FePAH}]^{+}$ and $\left[\mathrm{Fe}(\mathrm{PAH})_{2}\right]^{+}$complexes, found to be in line with the AIBs, with additional specific features in the far-IR range and -(ii)- the photoevaporation of $\left[\mathrm{Fe}_{x} \mathrm{PAH}_{y}\right]^{+}$clusters as possible candidates for very small grains releasing PAHs in photodissociation regions. These results call for new astronomical data at high spatial resolution. Studies on larger clusters will require further experimental and theoretical developments.
\end{abstract}

\section{Introduction: The Fe-PAH proposal}

Serra et al. (1992) and Chaudret et al. (1991) first proposed that $\pi$-adducts of polycyclic aromatic hydrocarbons (PAHs) and Fe atoms are stable species that can form efficiently in the conditions of the interstellar medium (ISM) and play a role in its chemistry. The efficient coordination of Fe atoms on PAHs would then partially account for the significant depletion of elemental Fe from the interstellar gas-phase. Besides, Fe atoms or clusters trapped on carbonaceous grains could play a catalytic role in the formation of large hydrocarbons, and of PAHs in particular.

\footnotetext{
1 Université de Toulouse, UPS, LCPQ (Laboratoire de Chimie et Physique Quantiques), IRSAMC, 118 Route de Narbonne, 31062 Toulouse, France

2 CNRS, LCPQ, IRSAMC, 31062 Toulouse, France

3 Université de Toulouse, UPS, Centre d'Étude Spatiale des Rayonnements (CESR), Observatoire Midi-Pyrénées, 9 AV. Colonel Roche, 31028 Toulouse Cedex 04, France

4 CNRS, CESR, 31028 Toulouse, France
} 
This inital proposal opened a new class of species to be considered and motivated at the time a few experimental (Marty 1996; Marty et al. 1996) and theoretical (Klotz et al. 1995) studies, along with astrophysical modeling (Marty et al. 1994; Ristorcelli \& Klotz 1997).

It has recently been reconsidered in the new astrophysical context e.g.: -(i)- the search for the carriers of the blue component of the $6.2 \mu \mathrm{m}$ aromatic infrared band (AIB) assigned to PAH-derived species (Peeters et al. 2002) such as substituted PAHs like PANHs ${ }^{+/ 0 /-}$ (Bauschlicher et al. 2009; Hudgins et al. 2005), or PAHs coordinated to an heteroatom such as Mg (Bauschlicher 2009; Bauschlicher \& Ricca 2009), Si (Joalland et al. 2010, 2009) or Fe (Simon \& Joblin 2007, 2010).

-(ii)- the search for candidates for the VSGs located in molecular clouds and shown to release PAH molecules at the surface of the cloud (Rapacioli, see elsewhere in this volume): the presence of heteroatoms like $\mathrm{Fe}$ (or $\mathrm{Si}$ ) could stabilize $\mathrm{PAH}$ clusters, that are possible candidates for these VSGs (Rapacioli et al. 2005). The experimental study, coupled with theoretical modeling, of the photostability of $\left[\mathrm{Fe}_{x} \mathrm{PAH}_{y}\right]^{+}$clusters in the conditions of the ISM, indeed shows that such clusters can be good candidates for these VSGs (Simon \& Joblin 2009).

\section{Stability of $[\mathrm{FePAH}]^{+}$and $\left[\mathrm{Fe}_{\mathrm{x}} \mathrm{PAH}_{\mathrm{y}}\right]^{+}$in the ISM}

Calculations based on Density Functional Theory (DFT) show that $[\mathrm{FePAH}]^{+}$ complexes are intrinsically more stable than $[\mathrm{FePAH}]^{0}$ complexes due to larger Fe-PAH binding energies, e.g. $\sim 2.5 \mathrm{eV}$ for $[\mathrm{FePAH}]^{+}$complexes $v s . \sim 0.6 \mathrm{eV}$ for $[\mathrm{FePAH}]^{0}$ complexes (Simon \& Joblin 2007). They are thus more likely to survive in the conditions of the ISM than their neutral counterparts. $\left[\mathrm{FeC}_{24} \mathrm{H}_{12}\right]^{+}$ and $\left[\mathrm{Fe}_{x}\left(\mathrm{C}_{24} \mathrm{H}_{12}\right)_{2}\right]^{+}(\mathrm{x}=1,4)$ complexes were formed in the PIRENEA set-up, a cold ion trap dedicated to astrochemistry (Simon \& Joblin 2009). Interestingly, complexes with dehydrogenated coronene also form when the number of $\mathrm{Fe}$ atoms reaches 3 , suggesting the occurence of intramolecular organometallic reactions. Each $\left[\mathrm{FeC}_{24} \mathrm{H}_{12}\right]^{+}$and $\left[\mathrm{Fe}_{x}\left(\mathrm{C}_{24} \mathrm{H}_{12}\right)_{2}\right]^{+}(\mathrm{x}=1,3)$ was isolated and photodissociated under soft irradiation conditions. The major dissociation pathway is a sequential loss of $\mathrm{Fe}$ atoms from $\left[\mathrm{Fe}_{3}\left(\mathrm{C}_{24} \mathrm{H}_{12}\right)_{2}\right]^{+}$and $\left[\mathrm{Fe}_{2}\left(\mathrm{C}_{24} \mathrm{H}_{12}\right)_{2}\right]^{+}$to lead to $\left[\mathrm{Fe}\left(\mathrm{C}_{24} \mathrm{H}_{12}\right)_{2}\right]^{+}$that loses one coronene molecule under further irradiation. The smallest complex $\left[\mathrm{FeC}_{24} \mathrm{H}_{12}\right]^{+}$then loses $\mathrm{Fe}$, leaving $\left[\mathrm{C}_{24} \mathrm{H}_{12}\right]^{+}$as the final charged photofragment. The stochiometry of these $\left[\mathrm{Fe}_{x}\left(\mathrm{C}_{24} \mathrm{H}_{12}\right)_{2}\right]^{+}$complexes and their dissociation sequence make them good candidates for the VSGs mentioned in the previous section. The photodissociation kinetics of the smallest complex $\left[\mathrm{FeC}_{24} \mathrm{H}_{12}\right]^{+}$was simulated using a Monte Carlo kinetic model in order to retrieve, from the fit of the experimental points, data such as the dissociation pre-exponential factor $A_{d}=10^{12} \mathrm{~s}^{-1}$ and the mean internal energy at dissociation $\left.\left(<U_{d}\right\rangle_{\min } \sim 5 \mathrm{eV}\right)$. These data can now be inserted into astrochemical models. 


\section{Mid- and far-IR features of $[\mathrm{FePAH}]^{+}$and $\left[\mathrm{Fe}(\mathrm{PAH})_{2}\right]^{+}$complexes}

The AIBs are emission features from UV-excited species. Anharmonic effects are therefore expected to play an important role in these spectra. A laboratory approach to study these effects is to perform infrared multiple photon dissociation (IRMPD) spectroscopy experiments (Oomens, see elsewhere in this volume). This technique was applied to $\left[\mathrm{FeX} \mathrm{X}_{1,2}\right]^{+}$complexes with small PAHs $\left(\mathrm{X}=\mathrm{C}_{6} \mathrm{H}_{6}, \mathrm{C}_{10} \mathrm{H}_{8}\right.$, $\mathrm{C}_{13} \mathrm{H}_{10}$ (Szczepanski et al. 2006)) and to $\left[\mathrm{YFeC}_{24} \mathrm{H}_{12}\right]^{+}$heterogeneous complexes $\left(\mathrm{Y}=\mathrm{C}_{5} \mathrm{H}_{5}, \mathrm{C}_{5}\left(\mathrm{CH}_{3}\right)_{5}\right.$ (Simon et al. 2008)). To our knowledge, no IRMPD experiments have been performed yet on $\left[\mathrm{Fe}_{x} \mathrm{PAH}_{y}\right]^{+}$complexes with large PAHs, the production and dissociation of these species being challenging tasks. Infrared (IR) spectra of neutral FePAH complexes were recorded in cold rare-gas matrices (Elustondo et al. 1999; Wang et al. 2007). All laboratory spectra have been compared with calculated harmonic IR spectra obtained at the DFT level and the calculations have been extended to larger systems (Simon \& Joblin 2010). These calculations remain the most straightforward approach to test the contribution of various PAH-derived species to the AIBs.

\subsection{Harmonic spectra}

Using DFT calculations, we found that the $\pi$-coordination of an Fe atom on an individual $\mathrm{PAH}^{+}$leads to a collapse of the band intensity in the $6.2 \mu \mathrm{m}$ region where the CC stretching modes $\left(\nu_{C C}\right)$ are located - with respect to the $11.2 \mu \mathrm{m}$ region - where the intense $\mathrm{C}-\mathrm{H}$ out-of-plane bending modes $\left(\gamma_{\mathrm{CH}}\right)$ are found. A blueshift of the $\nu_{C C}$ band and a slight redshift of the $\gamma_{C H}$ band upon coordination of an Fe atom were also put foward (Simon \& Joblin 2007). Considering a mixture of cationic PAHs ranging from pyrene $\mathrm{C}_{16} \mathrm{H}_{10}$ to circumcoronene $\mathrm{C}_{54} \mathrm{H}_{18}$ (Simon \& Joblin 2010), the $\pi$-coordination of an Fe atom was shown to lead to:

-(i)- an increase of the intensity ratio of the $\mathrm{C}-\mathrm{H}$ stretching $\left(\nu_{C H}\right.$ at $\left.3.25 \pm 0.01 \mu \mathrm{m}\right)$ and $\gamma_{C H}$ bands with respect to the intense $\nu_{C C}$ band, and shifts of the $\nu_{C C}$ and $\gamma_{C H}$ band positions $(-0.1 \mu \mathrm{m}$ and $+0.04 \mu \mathrm{m}$ respectively), with characteristic profiles displaying a steep blue rise and an extended red tail,

-(ii)- the occurence of many new bands in the far-IR range. Some vibrational modes of the PAH skeleton are indeed activated due to symmetry reduction and new modes involving the motion of the Fe atom appear. In particular, an accumulation point due to the activation of the Fe-PAH stretching mode is observed at $\sim 40 \mu \mathrm{m}$. This range is thus suggested to contain the spectral fingerprint for the presence of $[\mathrm{M}-\mathrm{PAH}]^{+}(\mathrm{M}=\mathrm{Fe}, \mathrm{Si}, \mathrm{Mg})$ complexes in the ISM. Additional features in the [60-300] $\mu \mathrm{m}$ range were also found for complexes with large PAHs.

$\left[\mathrm{Fe}(\mathrm{PAH})_{2}\right]^{+}$were shown to be stable in low-temperature and low-pressure conditions (Simon \& Joblin 2009). The harmonic spectra of mixtures of homogeneous $\left[\mathrm{Fe}(\mathrm{PAH})_{2}\right]^{+}$and heterogeneous $\left[\left(\mathrm{C}_{66} \mathrm{H}_{20}\right) \mathrm{FePAH}\right]^{+}$for 6 compact PAHs ranging from pyrene $\left(\mathrm{C}_{16} \mathrm{H}_{10}\right)$ to circumovalene $\left(\mathrm{C}_{66} \mathrm{H}_{20}\right)$, were computed at the DFT level of theory (Fig. 1). Both mid-IR spectra present very similar features, making them 
a prototype for $\left[\mathrm{Fe}(\mathrm{PAH})_{2}\right]^{+}$complexes with large PAHs. The $6-9 \mu \mathrm{m}$ region differs from that of $[\mathrm{FePAH}]^{+}$complexes (Simon \& Joblin 2010), with an enhanced intensity of the $7.38 \mu \mathrm{m}$ band in particular. The intensity of the $3.25 \mu \mathrm{m}$ band is also considerably enhanced with respect to that of the $\gamma_{C H}$ and $\nu_{C C}$ bands. In the far-IR range, bands with reasonable intensities appear at $\sim 30$ and $100 \mu \mathrm{m}$. They are assigned respectively to the Fe-PAH stretching mode coupled to PAH-skeleton deformations, and to the Fe motion parallel to the PAH surface (Simon \& Joblin, in preparation).

The mid-IR spectra of $\left[\mathrm{Fe}(\mathrm{PAH})_{x}\right]^{+}(\mathrm{x}=1,2)$ are found to be good candidates to account for both positions and profiles of the AIBs. Their rich far-IR spectra call for new astronomical data from space missions such as the Herschel Space Observatory and the future SPICA telescope, that can probe this spectral range with an increased sensitivity.

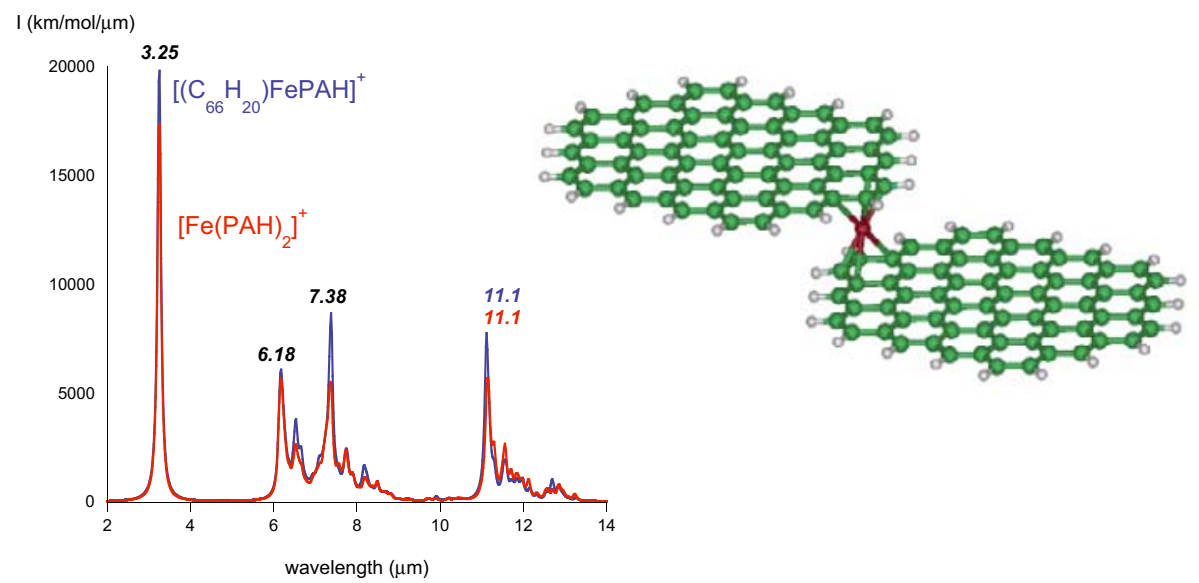

Fig. 1. Left: computed mid-IR harmonic spectra of mixtures of $\left[\mathrm{Fe}(\mathrm{PAH})_{2}\right]^{+}$(red) and $\left[\left(\mathrm{C}_{66} \mathrm{H}_{20}\right) \mathrm{FePAH}\right]^{+}$(blue) complexes. Right: stable structure of $\left[\mathrm{Fe}\left(\mathrm{C}_{66} \mathrm{H}_{20}\right)_{2}\right]^{+}$obtained at the DFT level of theory.

\subsection{Anharmonic effects on the mid-IR spectra of $[\mathrm{FePAH}]^{+}$}

The anharmonic effects in the mid-IR spectra of various $\mathrm{PAH}, \mathrm{PAH}^{+}$and $[\mathrm{SiPAH}]^{+}$ complexes were obtained from classical molecular dynamics (MD) simulations (Joalland et al. 2010) on a potential energy surface described at the DFTB (Density Functional Tight Binding) level of theory (Elstner et al. 1998). Using a similar approach, we performed a comparative study of the anharmonic effects on the IR spectra of $\left[\mathrm{FeC}_{24} \mathrm{H}_{12}\right]^{+}$and $\left[\mathrm{C}_{24} \mathrm{H}_{12}\right]^{+}$(Simon et al. submitted), showing in particular that:

-(i)- the anharmonicity of the $\gamma_{C H}$ band is decreased upon coordination of an $\mathrm{Fe}$ atom, resulting in the merging of the $\gamma_{C H}$ bands of $\left[\mathrm{FeC}_{24} \mathrm{H}_{12}\right]^{+}$and $\left[\mathrm{C}_{24} \mathrm{H}_{12}\right]^{+}$ at $\sim 700 \mathrm{~K}$ (Fig. 2), 
-(ii)- the anharmonicity of the $\nu_{C C}$ band is also decreased, enhancing the blueshift of the $\nu_{C C}$ band of $\left[\mathrm{FeC}_{24} \mathrm{H}_{12}\right]^{+}$with respect to that of $\left[\mathrm{C}_{24} \mathrm{H}_{12}\right]^{+}$.

The band positions were shown to have a linear dependence on temperature. Extrapolating these dependences to the mixture of $[\mathrm{FePAH}]^{+}$complexes with large PAHs studied by Simon \& Joblin (2010), we found that the $\gamma_{C H}$ band shifts from $11.02 \mu \mathrm{m}$ at $0 \mathrm{~K}$ to $11.23 \mu \mathrm{m}$ at $800 \mathrm{~K}$ whereas the $\nu_{C C}$ band shifts from $6.16 \mu \mathrm{m}$ to $6.31 \mu \mathrm{m}$. These results show that the assignment of the $6.2 \mathrm{vs} .6 .3 \mu \mathrm{m}$ components of the AIBs should be done with caution.

MD simulations also show that the diffusion of the Fe atom on the $\left[\mathrm{C}_{24} \mathrm{H}_{12}\right]^{+}$surface is observed at $\mathrm{T} \sim 850 \mathrm{~K}, e . g$. for an energy excess of $\sim 4 \mathrm{eV}$, which is below the dissociation energy determined to be $5 \mathrm{eV}$ (cf. Sect. 2). This DFTB/MD method also allows us to probe high-temperature chemistry and will be used for reactivity studies in a very near future.
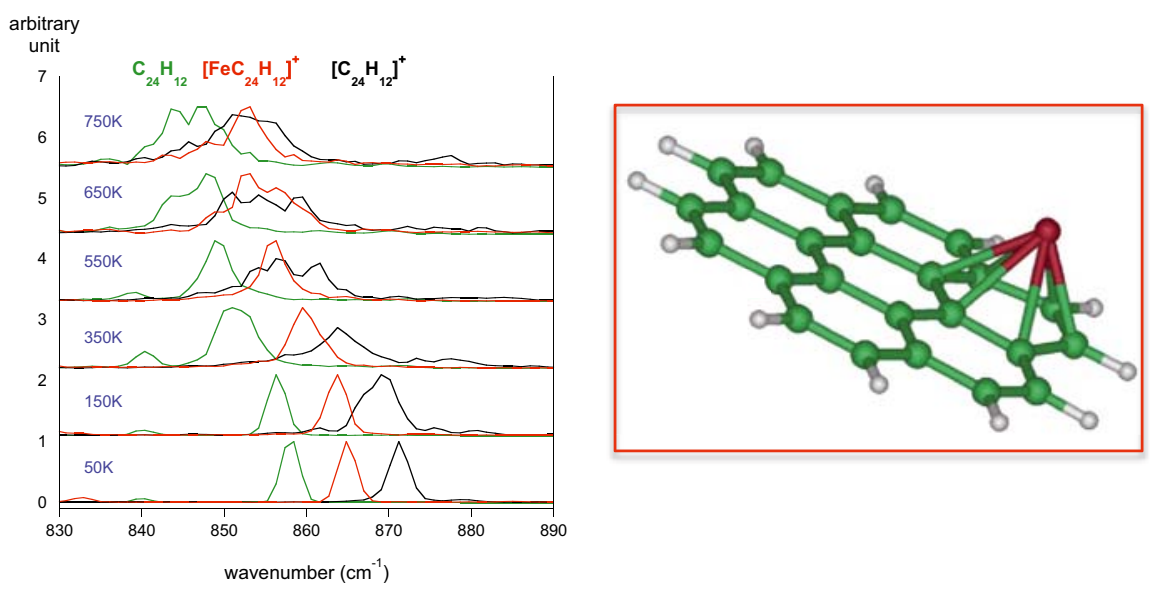

Fig. 2. Left: DFTB/MD simulated anharmonic IR spectra (zoom on the CH out-of-plane bending mode $\left.\gamma_{C H}\right)$ of $\left[\mathrm{FeC}_{24} \mathrm{H}_{12}\right]^{+}$(red) and $\left[\mathrm{C}_{24} \mathrm{H}_{12}\right]^{0 /+}$ (green/black). Right: most stable structure of $\left[\mathrm{FeC}_{24} \mathrm{H}_{12}\right]^{+}$obtained at the DFT level of theory.

\section{Conclusions and future work}

The computed harmonic mid-IR spectra of $[\mathrm{FePAH}]^{+}$and $\left[\mathrm{Fe}(\mathrm{PAH})_{2}\right]^{+}$complexes with large PAHs show that these species could contribute to the AIBs. The far-IR spectra of these complexes present specific features that need to be searched for in astronomical spectra. Experimental results show that $\left[\mathrm{Fe}_{x} \mathrm{PAH}_{y}\right]^{+}$complexes are likely candidates for astronomical VSGs releasing PAHs at the surface of molecular clouds. These studies have benefited from recent experimental and theoretical developments that will be pursued to improve our knowledge of larger clusters. Future work will include the study of the stability and spectroscopy of complexes of Fe atoms with dehydrogenated PAHs and the investigation of the role of FePAH complexes in the formation of $\mathrm{H}_{2}$. 


\section{References}

Bauschlicher, C.J., 2009, Mol. Phys., 107, 809

Bauschlicher, C.W., Peeters, E., \& Allamandola, L.J., 2009, Astrophys. J., 697, 311

Bauschlicher, C.W., Jr., \& Ricca, A., 2009, Astrophys. J., 698, 275

Chaudret, B., Le Beuze, A., Rabaa, H., Saillard, Y., \& Serra, G., 1991, New J. Chem., 15,791

Elstner, M., Porezag, D., Jungnickel, G., et al., 1998, Phys. Rev. B, 58, 11, 7260

Elustondo, F., Dalibart, M., Derouault, J., \& Mascetti, J., 1999, Phys. Chem. Earth C, 24,583

Hudgins, D.M., Bauschlicher, C.W., \& Allamandola, L.J., 2005, Astrophys. J., 632, 1, 316

Joalland, B., Rapacioli, M., Simon, A., et al., 2010, J. Phys. Chem. A, 114, 5846

Joalland, B., Simon, A., Marsden, C.J., \& Joblin, C., 2009, Astron. Astrophys., 494, 969

Klotz, A., Marty, P., Boissel, P., et al., 1995, Astron. Astrophys., 304, 520

Marty, P., 1996, Chem. Phys. Lett., 256, 669

Marty, P., de Parseval, P., Klotz, A., Serra, G., \& Boissel, P., 1996, Astron. Astrophys., 316,270

Marty, P., Serra, G., Chaudret, B., \& Ristorcelli, I., 1994, Astron. Astrophys., 282, 916

Peeters, E., Hony, S., Van Kerckhoven, C., et al., 2002, Astron. Astrophys., 390, 1089

Rapacioli, M., Joblin, C., \& Boissel, P., 2005, Astron. Astrophys., 429, 193

Ristorcelli, I., \& Klotz, A., 1997, Astron. Astrophys., 317, 962

Serra, G., Chaudret, B., Saillard, Y., et al., 1992, Astron. Astrophys., 260, 489

Simon, A., \& Joblin, C., 2007, J. Phys. Chem. A, 111, 9745

Simon, A., \& Joblin, C., 2009, J. Phys. Chem. A, 113, 4878

Simon, A., \& Joblin, C., 2010, Astrophys. J., 712, 69

Simon, A., \& Joblin, C., in preparation

Simon, A., Joblin, C., Polfer, N., \& Oomens, J., 2008, J. Phys. Chem. A, 112, 8551

Simon, A., Rapacioli, M., Lanza, M., Joalland, B., \& Spiegelman, F., Phys. Chem. Chem. Phys., submitted

Szczepanski, J., Wang, H., Vala, M., et al., 2006, Astrophys. J., 646, 666

Wang, Y., Szczepanski, J., \& Vala, M., 2007, Chem. Phys., 342, 107 